\title{
Parent-Child Nonverbal Engagement During Read Versus Sung Book-Sharing in Preschoolers with and without ASD
}

Talia Liu ${ }^{1}$, Benjamin G. Schultz ${ }^{2}$, Danielle Dai ${ }^{1}$, Christina Liu ${ }^{1}$, and Miriam D. Lense ${ }^{1,3,4}$

${ }^{1}$ Department of Otolaryngology-Head and Neck Surgery, Vanderbilt University Medical Center

${ }^{2}$ Department of Neuropsychology \& Psychopharmacology, Maastricht University ${ }^{3}$ Vanderbilt Kennedy Center, Vanderbilt University Medical Center ${ }^{4}$ The Curb Center for Art, Enterprise, and Public Policy, Vanderbilt University

\section{Manuscript in press at:}

Liu, T., Schultz, B. G., Dai, D., Liu, C., \& Lense, M. D. (2021). Parent-child nonverbal engagement during read versus sung book-sharing in preschoolers with and without ASD. Psychology of Music. https://doi.org/10.1177/03057356211058781

\section{Author Note}

Talia Liu (D) https://orcid.org/0000-0003-3534-9758

We have no known conflicts of interest to disclose. Correspondence concerning this article should be addressed to Talia Liu, Department of Otolaryngology, 1215 21st Avenue South, Room 10267, Medical Center East, South, Nashville, TN, 37232. Email: 
PARENT-CHILD NONVERBAL ENGAGEMENT

\section{Funding Acknowledgments}

The author(s) disclosed receipt of the following financial support for the research, authorship, and/or publication of this article: Research reported in this publication was supported in part by awards from the National Endowment for the Arts (1844332-38-C-18). The content is solely the responsibility of the authors and does not necessarily represent the official views of the National Endowment for the Arts (NEA). The NEA does not guarantee the accuracy or completeness of the information included in this report and are not responsible for any consequence of its use. This work was additionally supported by the Program for Music, Mind \& Society at Vanderbilt (with funding from the Trans-Institutional Programs Initiative), the VUMC Faculty Research Scholars Program, and the VUMC Department of Otolaryngology. Data collection and management used REDCap electronic data tools at Vanderbilt (UL1 TR000445 from NCATS/NIH). 
PARENT-CHILD NONVERBAL ENGAGEMENT

\begin{abstract}
Providing natural opportunities that scaffold interpersonal engagement is important for supporting social interactions for young children with Autism spectrum disorder (ASD). Musical activities are often motivating, familiar, and predictable, and may support both children and their interaction partners by providing opportunities for shared social engagement. We assessed multiple facets of nonverbal social engagement - child and caregiver visual attention and interpersonal movement coordination - during musical (song) and non-musical (picture) booksharing contexts in caregiver-child dyads of preschoolers with $(n=13)$ and without $(n=16)$ ASD. Overall, children with ASD demonstrated reduced visual attention during the book sharing activity, as well as reduced movement coordination with their caregivers, compared to children with typical development. Children in both diagnostic groups, as well as caregivers, demonstrated greater visual attention (gaze toward the activity and/or social partner) during song books compared to picture books. Visual attention behavior was correlated between children and caregivers in the ASD group but only in the song book condition. Findings highlight the importance of considering how musical contexts impact the behavior of both partners in the interaction. Musical activities may support social engagement by modulating the behavior of both children and caregivers.
\end{abstract}

Keywords: Autism spectrum disorders, parent-child interaction, song, attention, movement coordination 
PARENT-CHILD NONVERBAL ENGAGEMENT

\section{Parent-Child Nonverbal Engagement During Read Versus Sung Book-Sharing in Preschoolers with and without ASD}

Providing natural opportunities to scaffold young children's social engagement is important for supporting their social interactions, including for children with autism spectrum disorder (ASD) who have difficulties with social interaction and communication (American Psychiatric Association, 2013). Some activities may be more effective than others for supporting interpersonal engagement in children with ASD, such as structured activities that follow the child's focus of attention (Wimpory et al., 2007). Activities that follow the child's focus provide opportunities for interaction partners (such as caregivers) to be available for and responsive to the child, which supports children's developmental outcomes (Gulsrud et al., 2016; Landry et al. 2006). Empirical and theoretical reports suggest that musical activities might provide a natural context to foster engagement in young children with ASD as the familiarity and predictability of musical activities may support the behavior of both the child and the interaction partner, though the mechanisms underlying this effect are still under investigation (Hernandez-Ruiz, 2019; Kern et al., 2007; Kim et al., 2008; Lense \& Camarata, 2020; Simpson et al., 2013; Wimpory et al., 2007). For example, musical activities may support social attention, social bonding, and social coordination through involvement of the endogenous opioid and reward processing systems (Kokal et al., 2011; Matthew et al., 2020; Savage et al., 2020; Tarr et al., 2014). However, little research has considered how musical contexts modulate specific behaviors of the child and their adult interaction partner. In the current study, we examined whether musical activities impact attention and coordination in children with ASD and without ASD and their caregivers when sharing books together.

\section{Musical Activities and Shared Attention}




\section{PARENT-CHILD NONVERBAL ENGAGEMENT}

\section{Musical Activities and Visual Attention}

Attention to the eyes of another is an important form of social attention, which facilitates opportunities for joint attention (coordinated gaze between another person and referent) and has downstream effects on social cognition (Stephenson et al., 2021). However, reduced attention to the eyes of others and impairments in joint attention are common in ASD (Baron-Cohen, 1989; Costantino et al., 2017; Mundy, 2018; Sasson \& Touchstone, 2014). Musical activities may support engagement by increasing children's attention to the task and their social partner. Studies of children with typical development (TD) demonstrate that children increase their gaze toward an adult social partner during musical play and joint singing versus non-musical play (Beck \& Reiser, 2020) and increase their eye contact and mutual smiling toward another child during a synchronous rhythmic interaction game versus a nonsynchronous version (Tunçgenç \& Cohen, 2018). Several studies report increased engagement and attention during musical activities in children with ASD in therapeutic or educational settings where the child is interacting with a trained professional (Kim et al., 2008; Paul et al., 2015; Simpson et al., 2013). For example, young children with ASD had increased eye gaze and turn-taking with therapists during music therapy versus play therapy (Kim et al., 2008), increased eye contact and social gesture responses to a therapist's sung versus spoken prompts (Paul et al., 2015), increased eye contact toward others in a music therapy group versus non-musical social skills group (LaGasse, 2014), and increased task engagement during sung versus spoken directions in a receptive language learning activity (Simpson et al., 2013). In an eye-tracking study, children with ASD looked more at someone's face when the person was singing compared to when they were reading a book (Thompson \& Abel, 2018). The increased social attention during music therapy and musical activities occurs spontaneously and thus contrasts with therapeutic techniques that force 


\section{PARENT-CHILD NONVERBAL ENGAGEMENT}

eye contact, which may be uncomfortable and stressful for individuals with ASD (Hadjikhani et al., 2017; McGlensey, 2016). Therefore, musical contexts may provide an ecologically-valid, enjoyable context that naturally motivates social attention, including through eye contact, for children with TD and children with ASD.

\section{Musical Activities and Movement Coordination}

Musical activities may also support social interaction by enabling individuals to coordinate their movements with each other (Lense \& Camarata, 2020). Interpersonal coordination (i.e., social interaction behaviors aligned by time and form (McNaughton \& Redcay, 2020)) is an important component of social development (Stern, 1974; Trevarthen \& Aitken, 2001), and joint movement in musical activities are associated with prosocial behavior in children with TD (Trainor \& Cirelli, 2015). For example, preschoolers with TD who participated in a shared music play activity involving synchronous movements were more cooperative in a subsequent helping task than children participating in a parallel non-musical play activity (Kirschner \& Tomasello, 2009). Infants who were bounced synchronously with the movements of an unfamiliar adult while listening to music engaged in more helping behavior toward the adult than infants who were bounced asynchronously to the movements of the adult (Cirelli et al., 2014).

Compared to children with TD, children with ASD demonstrate reduced movement synchronization during activities in which interpersonal coordination is intentional (i.e., the goal of the activity is explicitly to synchronize) or spontaneous (i.e., the synchrony/coordination occurs without instruction) (Fournier et al., 2010; McNaughton \& Redcay, 2020; Zampella et al., 2020). Impaired interpersonal synchrony has been proposed as a potential early marker of ASD (Leclère et al., 2014). For example, school-aged children with ASD demonstrated reduced 


\section{PARENT-CHILD NONVERBAL ENGAGEMENT}

movement synchronization with an adult experimenter than children with TD during a simultaneous imitation task and an interpersonal clapping game (Fitzpatrick et al., 2013, 2017). In another study, parents read a story book to their preschool-aged child while both the parent and child sat in separate rocking chairs (Marsh et al., 2013). Preschoolers with ASD exhibited less spontaneous in-phase rocking with their parent's rocking than preschoolers with TD (Marsh et al., 2013).

Parents are important and motivating social partners in their children's lives, including during musical activities (Politimou et al., 2018), yet little is known about how musical contexts facilitate parent-child interactions in children with ASD. Most research has focused on the child's behavior during musical activities. However, aspects of shared musical activities such as their familiarity, predictability, and emotionality, may also support parents' behaviors and receptiveness to moments of shared engagement with their children (Hernandez-Ruiz, 2020; Lense \& Camarata, 2020). Parents of young children with ASD reported that musical activities (e.g., family-centered music therapy) provided an interactive context in which they could share a play experience with their children (Thompson, 2018; Thompson et al., 2019). Parents of children with ASD also provided more physical play responses to their child during musical versus non-musical toy play (e.g., imitating child; Boorom et al., 2020). As parent responsiveness is linked to children's social communication development (e.g., Gulsrud et al., 2016), musical activities may provide a context conducive to parents being available for and responsive to their child in order to support their child's social engagement. A better understanding of how musical activities impact both children's and parents' behavior, engagement, and attention may have implications for incorporating these activities into natural play contexts and parent-child interventions. 
PARENT-CHILD NONVERBAL ENGAGEMENT

\section{Book Reading Activities and Shared Attention}

Shared book reading is a common joint parent-child activity that involves attention to one's partner and a common object (book). During shared book reading activities, preschoolers with ASD generally show greater passive engagement or non-engagement compared to sameaged peers with TD (Bean et al., 2019; Fleury \& Hugh, 2018; Lanter et al., 2013; Westerveld et al., 2020). However, children's engagement can be moderated by the specific book context and parents' behavior (Fleury \& Hugh, 2018). Both children with ASD and children with TD were more actively engaged (verbally/nonverbally communicating with their parents about the book) during familiar books compared to novel books (Fleury \& Hugh, 2018). Increased quality of parents' book reading (e.g., supporting child's attention to the text and reading comprehension) also increased active engagement in children with ASD and TD during a book sharing activity (Fleury \& Hugh, 2018). However, to the knowledge of the authors, no studies have considered joint book sharing in a musical compared to a non-musical context.

In the current study, we investigated how a musical context may modulate child and parent behavior during book sharing. We considered two measures of nonverbal engagement during parent-child musical and non-musical book sharing: Visual attention (gaze) and interpersonal movement coordination. We focused on these nonverbal measures of engagement due to their involvement in both musical and non-musical interactions (e.g., Kim et al., 2008; LaGasse, 2014; Paul et al., 2015; Tunçgenç \& Cohen, 2018) and because they can be used with children with varying language levels (Kim et al., 2008; Pfeiffer et al., 2018). These measures also allowed us to consider the behaviors of each partner individually as well as within an interactional, dyadic framework. 


\section{PARENT-CHILD NONVERBAL ENGAGEMENT}

With three aims, we address whether musical contexts increase visual attention and interpersonal coordination for parent-child dyads of children with TD and ASD. Our first aim was to compare caregiver and child nonverbal engagement during book sharing with versus without a musical context. In order to create these contexts, caregiver-child dyads shared children's picture (non-musical) books and song (musical) books. We hypothesized that, for both ASD and TD dyads, the musical context of song books would increase child and caregiver engagement as measured via increased visual attention to the activity and increased interpersonal movement coordination. The second aim was to compare caregiver and child nonverbal engagement during dyadic book sharing for dyads with children with ASD and dyads with children with TD. We hypothesized that children with ASD would show less engagement than children with TD during the book sharing activity. The third aim was to examine individual differences in engagement during these book sharing activities. We hypothesized that caregivers' and children's visual attention would positively correlate with each other, reflecting caregivers' attunement to their children's engagement in the task. We also hypothesized that children's engagement would relate to their language level.

\section{Method}

\section{Participants}

Participants included 29 preschool-aged children with ASD (male $n=10$, female $n=3$ ) aged between 21-69 months and with TD (male $n=10$, female $n=6$ ) aged between 20-52 months and one of their caregivers. In the ASD group, caregivers included 9 mothers, 2 fathers, 1 grandmother, and 1 grandfather; in the TD group, caregivers included 12 mothers, 3 fathers, and 1 grandmother. To encompass the grandparents, the term "caregiver" (rather than parent) will be used throughout the rest of the manuscript. Two additional participants (one ASD, one TD) were 
PARENT-CHILD NONVERBAL ENGAGEMENT

excluded from the study because the caregivers did not follow the task instructions (below; i.e., did not sing during the song book condition). Participants were recruited through medical center clinics, preschools, community events, and social media. No musical training was required for participation. The children with ASD and with TD were matched on nonverbal developmental level (Mullen Scales of Early Learning [MSEL] Visual Reception age equivalences (see Table

1). Children with ASD were older than children with TD and had lower language levels (MSEL Receptive/Expressive Language age equivalences (see Table 1).

\section{Table 1}

Child Demographics and Clinical Scores by Group

\begin{tabular}{|c|c|c|c|c|}
\hline & ASD & TD & $\begin{array}{c}\text { Test } \\
\text { Statistic } \\
\left(\chi^{2} \text { or } t\right)\end{array}$ & $\begin{array}{c}\text { Significance } \\
(p)\end{array}$ \\
\hline$n(\mathrm{M}: \mathrm{F})$ & $13(10: 3)$ & $16(10: 6)$ & 0.70 & 0.40 \\
\hline Chronological Age (months) & $46.6(10.3)$ & $35.9(8.5)$ & 3.06 & $0.005 * *$ \\
\hline Race (\% Caucasian) & $69.2 \%$ & $87.5 \%$ & 1.46 & 0.23 \\
\hline $\begin{array}{l}\text { Household Income }(\% \text { Over } \\
\$ 70,000)\end{array}$ & $69.2 \%$ & $67.8 \%$ & 0.001 & 0.98 \\
\hline MSEL VR T Score & $42.0(16.3)$ & $61.8(10.9)$ & -3.85 & $<0.001 * * *$ \\
\hline MSEL VR AE (months) & $38.4(14.7)$ & $43.6(13.9)$ & -0.94 & 0.35 \\
\hline MSEL RL/EL T Score (average) & $33.6(11.4)$ & $57.3(8.8)$ & -6.10 & $<0.001 * * *$ \\
\hline MSEL RL/EL AE (average; months) & $29.6(14.0)$ & $42.1(13.0)$ & -2.40 & $0.02 *$ \\
\hline ADOS-2 Comparison Score & $6.8(2.8)$ & - & - & \\
\hline
\end{tabular}


PARENT-CHILD NONVERBAL ENGAGEMENT

Note. Mean (SD); MSEL = Mullen Scales of Early Learning; VR = Visual Reception (nonverbal problem solving); RL/EL = Receptive Language/Expressive Language; $\mathrm{AE}=$ age equivalence (months). T-scores have mean $=50$, standard deviation $=10$.

Significance: ${ }^{*} p<.05 .{ }^{*} p p<.01 . * * * p<.001$

All children with ASD were diagnosed prior to study enrollment by licensed clinicians with research reliability on the Autism Diagnostic Observation Schedule (ADOS-2) (Lord et al., 2012). Twelve children with ASD and all children with $\mathrm{TD}^{1}$ were administered the MSEL, a standardized developmental assessment for infants and children ages birth to 68 months (Mullen, 1995). The MSEL measures skills in five domains: gross motor, fine motor, visual reception (nonverbal problem solving), receptive language, and expressive language. MSEL Language Composite (averages of MSEL Receptive Language and Expressive Language scores) was used as measures of children's language functioning.

The university Institutional Review Board (IRB) approved the study protocol. Written, informed consent was obtained by a parent or guardian of the children. Children above the age of three years and with appropriate language level provided verbal assent.

\section{Procedure}

\section{Book Sharing Activity}

The book sharing activity was completed as part of a larger task battery and was administered following other study activities; thus, all children and caregivers were familiar with the assessment room for the task. The caregiver-child dyad sat across from each other at a table in an assessment room at the university research center. Caregivers were given two bags of books and were verbally instructed to share books in the first bag for five minutes and then,

\footnotetext{
${ }^{1}$ MSEL was not administered for one child with ASD due to experimenter error; MSEL language subscales were not administered for one child with TD who was a dual language learner.
} 


\section{PARENT-CHILD NONVERBAL ENGAGEMENT}

following hearing a knock on the door, to share books in the second bag for five minutes. Caregivers were instructed to have only one book out on the table at a time but were not informed about the content of the books in each bag in advance. For video purposes (due to movement analyses), caregivers were instructed to try to keep their children and themselves on their respective sides of the table and to avoid touching their children when possible. When caregivers opened each bag, they saw an information sheet, which instructed them to "read and share" (picture book bag) or to "sing and share" (song book bag) and also provided reminders about the activity length and positioning at the table. The first bag contained five wordless picture books (e.g., Good Dog Carl [Day, 2009]; Mine! [Crum, 2012]), and the second bag contained five song books (e.g., Wheels On The Bus [Raffi, 1988]; You Are My Sunshine [Davis, 2011]). Due to concerns about losing children's attention and transitioning if the song book activity were completed first, the picture book activity was completed first for all participants. Following completion of the task, caregivers were asked if they knew any of the books or songs. Caregivers reported that they were not familiar with the exact picture or song books but that they knew the associated songs for the song books they shared. This was also evidenced through observation of the caregivers singing the songs in the song book condition as caregivers could not sing songs they did not know.

The book sharing interaction was recorded using two video cameras recording at $30 \mathrm{~Hz}$. The primary camera angle captured the profile view of both the caregiver and child, and a secondary camera angle captured the frontal view of the child.

\section{Visual Attention Coding ( $V A C)$}

Caregivers' and children's visual attention (gaze) during the book sharing activity were manually coded from videos using a five-second, hierarchical partial interval coding schema 


\section{PARENT-CHILD NONVERBAL ENGAGEMENT}

adapted from Klimenko (2007). Coding began when the title of the book was said or the book was opened, whichever came first. Each five-second interval was coded as either Book sharing or Transition (changing between books); VAC means were calculated only from the Book sharing intervals. On average, 4.70 minutes $(S D=0.27)$ were coded for the picture book condition and 4.37 minutes $(S D=0.44)$ were coded for the song book condition for VAC scores. The codes for visual attentional behavior for the child ranged from 0 (looking away from book and caregiver) to 4 (looking at caregiver for at least two seconds, i.e., sustained attention to caregiver) for each five-second interval. For caregivers, the codes ranged from 0 (looking away from book and child) to 5 (looking at child for at least two seconds, i.e., sustained attention to child). The coding scheme takes into account that during a triadic interaction, it is appropriate for an individual to direct their attention toward both their partner and the shared object, and also considers whether the visual attention is for a short moment (glance) or if it is sustained. The different coding schema (and ranges) for child and caregiver capture differences in ways young children and adult caregivers engage in this interactive task, reflecting caregivers' more advanced and varied approaches to coordinating gaze and shifting attention during book sharing. See Table 2 for a full description of the coding schema. Mean scores for each book condition were calculated for each child and caregiver. Coding occurred using the primary camera angle (capturing profile view of caregiver and child). If a child's gaze was unclear, coders could defer

to the secondary camera angle. Videos were coded using ProcoderDVTM (Tapp \& Walden, 1993) and were divided among three trained Research Analysts. A second coder co-coded 17\% of the videos for reliability (child visual attention $\mathrm{ICC}=0.91$; caregiver visual attention $\mathrm{ICC}=0.92$ ).

\section{Table 2}


PARENT-CHILD NONVERBAL ENGAGEMENT

Visual Attention Coding (VAC) Schema (adapted from Klimenko, 2007)

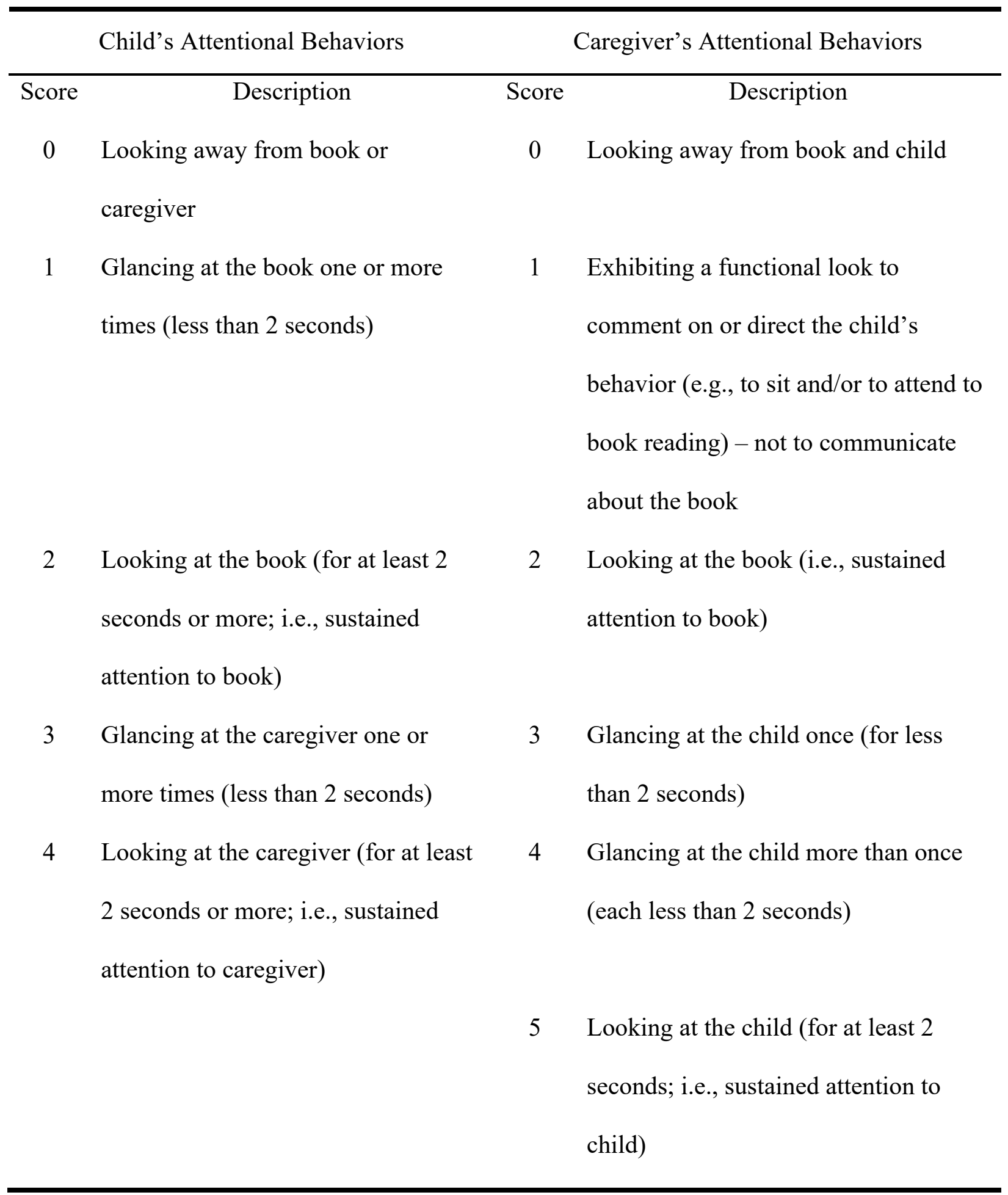




\section{PARENT-CHILD NONVERBAL ENGAGEMENT}

Using the profile camera view of the dyad, separate regions of interest (ROIs) were defined for the caregiver and child based on their positioning across from each other. In order to be used for analysis of interpersonal coordination, excerpts could not contain any ROI crossings (i.e., participants had to be on their respective sides of the table) or automatic camera refocusing. For these reasons, one-minute excerpts of each participant's picture book and song book activities were extracted for analysis starting at least 15 seconds after the book sharing began. The first one-minute excerpt that met these criteria was extracted. If no section with a oneminute duration met the criteria existed, the next longest possible excerpt (at least 30 seconds) was extracted. Video excerpts only included time when the dyad was continuously sharing a book; they did not include any time when the dyad was transitioning between books. The excerpts for movement analyses averaged 58.1 seconds $(S D=6.8$, range $=31.8-60)$. Of the 58 excerpts, 50 reached the full one-minute duration.

To analyze coordinated movements within the dyad, we used time series analysis methods that have previously been used to examine coordination of speech and movement in musical and nonmusical tasks (e.g., Schultz et al., 2016; Schultz et al., 2021; Schultz \& Palmer, 2019). We extracted the magnitude of change between consecutive frames within the two ROIs containing only the caregiver and only the child (see Ramseyer, 2020). Videos were converted to grayscale then filtered in the temporal domain using a bandpass Butterworth filter between $1 \mathrm{~Hz}$ and $14 \mathrm{~Hz}$ to remove artifacts relating to frame changes, interlacing, and slow drifts. The magnitude of change between consecutive frames was extracted for each ROI. Each time series was range normalized and subjected to time series analysis. We used dynamic time warping on the two time series to temporally align movement patterns within a window of five seconds. Data were pre-whitened to remove autoregressive components following Dean and Dunsmuir (2016). 


\section{PARENT-CHILD NONVERBAL ENGAGEMENT}

This conservative approach reduces the risk of spurious significant cross-correlation coefficients although the observed coefficients may underestimate movement coordination. The crosscorrelation coefficient at lag 0 was calculated to measure the strength of the relationship between the movement of the caregiver and child. To allow cross-correlation coefficients to be compared between conditions, these values were Fisher transformed prior to statistical analysis.

\section{Data Analysis}

Separate linear mixed-effects models (LMEM) were used to analyze children's mean visual attention, caregivers' mean visual attention, and interpersonal movement coordination (Fisher transformed cross-correlation coefficients) during the book sharing activities. All LMEMs contained the fixed factors Book (2; Picture, Song; within-subjects) and Group (2; ASD, TD; between subjects), and the random factor Dyad where Book was nested within Dyad and allowed unequal variance between ASD and TD groups. Effect sizes were measured using generalized eta squared $\left(\eta^{2}\right)$ for repeated-measures effects, where $0.02=$ small, $0.13=$ medium, and $0.26=$ large (Bakeman, 2005). Spearman correlations $\left(r_{s}\right)$ were computed to assess children's and caregiver's mean visual attention across the picture and song book sharing activities. Spearman correlations were also used to characterize relationships between children's visual attention scores and MSEL Language age equivalents. Correlation coefficients were interpreted using Cohen's guidelines where $0.10=$ small, $0.30=$ medium, and $0.50=$ large (Cohen, 2013).

Videos were analyzed using custom-made scripts in MATLAB. Analyses were conducted using R Software (R Core Team, 2019). Correlations were performed using the cor function, LMEMs were performed using the lmer function from the lme4 library (Bates et al., 2015), and within-subject effect sizes were obtained using the aov_car function from the afex library 
PARENT-CHILD NONVERBAL ENGAGEMENT

(Singmann et al., 2020). Time series analysis and dynamic time warping were performed using the TSA (Chan et al., 2020; Cryer \& Chan, 2008) and $d t w$ (Giorgino, 2009) libraries, respectively.

\section{Results}

\section{Child Visual Attention}

For children's visual attention, there were significant main effects of Book, $F(1,27)=$ 8.68, $p=0.007, \eta^{2}{ }_{G}=0.05$ and Group, $F(1,27)=4.34, p=0.047, \eta^{2}{ }_{G}=0.12$, indicating small and medium effect sizes, respectively. There was no significant interaction between Book and Group, $F(1,27)=0.48, p=0.50, \eta G^{2}{ }_{G}=0.003$. As shown in Figure 1, children mostly visually attended to the books with slightly higher visual attention scores during the song book activity $(\mathrm{M}=2.19, \mathrm{SEM}=0.059)$ than during the picture book activity $(\mathrm{M}=2.07, \mathrm{SEM}=0.06)$.

Children with TD $(M=2.23, S E M=0.037)$ exhibited slightly higher visual attention scores than children with $\operatorname{ASD}(M=2.01, S E M=0.078)$.

\section{[Insert Figure 1]}

We also assessed the relationship between children's visual attention levels and children's language level for each group. Individual differences in children's visual attention were generally consistent across the picture and song book activities in children with ASD, $r_{s}(11)$ $=0.68, p=0.013$, and children with $\mathrm{TD}, r_{s}(14)=0.60, p=0.014$, both indicating large effect sizes. We therefore averaged children's visual attention scores across the picture and song book activities. For children with ASD, visual attention scores were significantly correlated with their MSEL Language age equivalents, $r_{s}(10)=0.67, p=0.018$, suggesting greater visual attention to the activity/caregiver in children with ASD with higher language skills. There was no significant 
PARENT-CHILD NONVERBAL ENGAGEMENT

relationship between children with TD's visual attention scores and language skills, $r_{s}(13)=-0.2$, $p=0.92$

\section{Caregiver Visual Attention}

For caregivers' visual attention, there was a significant main effect of Book, $F(1,27)=$ 21.69, $p<0.001, \eta^{2}{ }_{G}=0.16$, a medium effect size. As shown in Figure 2, caregivers of children with ASD and TD demonstrated greater visual attention scores, suggesting somewhat increased gaze toward their children, during song books $(M=3.73, S E M=0.096)$ than during picture books $(M=3.33, S E M=0.075)$. However, there was no significant main effect of Group, $F(1$, 27) $=0.05, p=0.82, \eta^{2}{ }_{G}=0.002$, or significant interaction between Book and Group, $F(1,27)=$ $0.19, p=0.67, \eta^{2}{ }_{G}=0.002$.

[Insert Figure 2]

\section{Visual Attention Within Dyads}

We also examined the relationships between child and caregiver visual attention for the song and picture book activities. There was a significant correlation with a large effect size between caregiver and child mean visual attention scores in dyads with children with ASD during the song book activity, $r_{s}(11)=0.72, p=0.007$ (Figure 3a). This relationship was not observed during the picture book activity, $r_{s}(11)=-0.14, p=0.66$. For the dyads with children with TD, caregivers' and children's mean visual attention scores were not significantly correlated during the song book activity, $r_{s}(14)=0.25, p=0.35$, or during the picture book activity, $r_{s}(14)=0.30, p=0.26($ Figure $3 b)$.

\section{[Insert Figure 3]}

\section{Interpersonal Movement Coordination}


PARENT-CHILD NONVERBAL ENGAGEMENT

For movement coordination, there was a significant main effect of Group, $F(1,54)=$ $8.05, p=0.006, \eta^{2}{ }_{G}=0.13$ (medium effect size), but no significant main effect of Book, $F(1,54)$ $=0.78, p=0.38, \eta^{2}{ }_{G}=0.01$, or interaction between Group and Book, $F(1,54)=0.08, p=0.78$, $\eta^{2}{ }_{G}=0.001$. As shown in Figure 4, while overall magnitude of coordination was moderate, movement within TD dyads (mean $r=0.37, S E M=0.017$ ) was more coupled than within ASD dyads (mean $r=0.31, S E M=0.013)$.

\section{[Insert Figure 4]}

\section{Discussion}

The current study examined caregiver-child engagement during shared book activities across multiple levels of analysis (visual attention, movement coordination) and contexts (picture and song books). Visual attention scores were higher in children with TD than in children with ASD. While all children predominantly focused their attention on the books, children with TD spent somewhat more time engaged in the activity than children with ASD by glancing at their caregivers. This supports our second hypothesis and is consistent with evidence that children with ASD show reduced preference for social stimuli and attend to faces less than children with TD (Sasson \& Touchstone, 2014). We observed no difference in caregivers' visual attention by children's diagnostic status (ASD, TD) during the book sharing activity. This finding is in line with previous observations showing the quality of caregivers' book reading does not differ between caregivers of children with ASD and TD (Fleury \& Hugh, 2018).

For both caregivers and children, visual attention scores were significantly greater during the song book activity than during the picture book activity, supporting our first hypothesis that musical activities create a context that scaffolds caregiver-child engagement. Overall, children's visual attention scores in both groups suggested that children's gaze was primarily directed 


\section{PARENT-CHILD NONVERBAL ENGAGEMENT}

toward the books. This is unsurprising given that the books were generally directly in front of the children and the task focus was on book sharing. Although the effect size was small, both children with ASD and TD showed slightly greater visual attention scores (i.e., more occasional gazing toward their caregiver and/or less looking away from the task) during the song books compared to picture books. As depicted in Figure 1, the range of visual attention scores between children with ASD and TD were similar in the song book condition while more children with ASD had lower visual attention scores than their TD peers in the picture book condition. Another recent study also reported that infants with and without ASD displayed similar amounts of gaze toward an adults' face when the adult engaged them with song but that infants with ASD gazed less at the adult when she engaged them with speech (Macari et al., 2020). Song and music contexts may naturally support children's engagement across a range of child functioning levels. Indeed, there is some suggestion that music therapy may be particularly appropriate for children with lower cognitive skills (Crawford et al., 2017).

Caregivers also exhibited more visual gaze toward their children during the song book activity than the picture book activity. Additionally, caregiver-child visual attention scores were correlated in the song book activity for the ASD group. Attributes of song activities, such as their familiarity and predictability, may be particularly relevant for modulating dyadic interactions in dyads with children with ASD. The slight increases in both child and parent attention for the song book context may suggest that caregivers are somewhat more available for moments of social engagement (e.g., for times when children do direct their gaze toward their caregivers) and to reinforce and support their children's social and on-task behavior. Future studies could examine if song contexts support engagement between caregiver-child dyads with ASD by modulating both partners' gaze behavior on a moment-to-moment basis in an interactive, 


\section{PARENT-CHILD NONVERBAL ENGAGEMENT}

contingent manner (i.e., via a transactional feedback loop), as well as examine if these effects would compound over time (e.g., with repeated sharing of the same song book).

Our findings are consistent with prior studies of increased social attention in children with TD or ASD during musical activities (e.g., Beck \& Reiser, 2020; Kim et al., 2008; LaGasse, 2014; Paul et al., 2015; Simpson et al., 2013; Tunçgenç \& Cohen, 2018) and also extend such findings to the behavior of caregivers engaging in these activities. Dyadic musical activities naturally involve familiarity, predictability, and emotionality, all of which may facilitate engagement (Cirelli et al., 2018; Hernandez-Ruiz, 2020; Lense \& Camarata, 2020). Previous studies indicate that familiarity with specific books increases engagement in both children with ASD and TD during shared book reading (Fleury \& Hugh, 2018). In the current study, although caregivers and children did not know the specific books, caregivers had to know the songs in order to sing the song books and caregivers indicated that their children knew the songs. Familiarity with the songs may have allowed caregivers to direct their gaze at their children more regularly. During the wordless picture books, caregivers may have needed to look at the pictures more to describe the pictures and narrate a story. This suggests that even in an activity with novel components (i.e., the specific books), known songs provide a context that may help structure caregivers' and their children's behavior. The predictable rhythmic patterns, melodies, and lyrics of the song books may help dyads track how the song unfolds over time and contribute to increased attention and behavior modulation (Hardy \& LaGasse, 2013; Miller et al., 2013). Song activities may also support attention and engagement because they naturally involve predictable and salient multimodal cues (e.g., head movements, gestures) that are coordinated with the rhythm of the song (Lense \& Jones, 2016; Trehub et al., 2016) and because song activities modulate emotion and arousal levels in both partners in the dyad (Cirelli et al., 2020; Cirelli \& 


\section{PARENT-CHILD NONVERBAL ENGAGEMENT}

Trehub, 2020; Fancourt \& Perkins, 2018; Shenfield et al., 2003). These attributes of the song books might have made them more accessible for children and caregivers.

This is the first study to examine caregiver-child body movement coordination in preschoolers during song and picture book sharing tasks. Our finding that movement of dyads with children with TD were more coordinated than dyads of children with ASD supports our second hypothesis and is in line with a growing body of research into reduced spontaneous interpersonal movement coordination in older children and adults with ASD (Fitzpatrick et al., 2017; Fournier et al., 2010) as well as reduced in-phase rocking behavior by children with ASD versus children with TD (Marsh et al., 2013). Decreased interpersonal coordination may reflect reduced attunement to the social interaction in dyads with children with ASD and may involve a combination of attentional, social, and motor processes (e.g., Fitzpatrick et al., 2017). While the overall magnitude of the movement coordination was small, this may in part reflect the free context of the activity (sharing books while sitting at a table) as prior studies of movement synchrony in children have generally used more explicit movement or imitation tasks (e.g., Fitzpatrick et al., 2017; Marsh et al., 2013). Moreover, our study used conservative estimates of coordination using pre-whitening in order to circumvent spurious correlations (following Dean \& Dunsmuir, 2016).

In contrast to our findings with visual attention coding, we did not observe any differences in movement coordination between the song and picture book sharing conditions. Although we are surprised by this finding, this may be explained by multiple factors. First, caregivers and children were seated at a table for these activities, potentially limiting the range of movements across the song and picture book contexts. Second, due to potential ROI crossings, only a shorter portion (up to one minute) of the interaction was analyzed for the movement 


\section{PARENT-CHILD NONVERBAL ENGAGEMENT}

analyses versus longer periods of time for the visual attention coding. Third, while we hypothesized increased coordination in the musical context due to the opportunity to utilize pantomime gestures and move to rhythmic cues, dyads were not required to engage in songassociated gestures during the activity. This is consistent with findings that young children may not spontaneously sustain rhythmic behavior during musical activities, especially in laboratory settings (Cirelli \& Trehub, 2019; Eerola et al., 2006; Endedijk et al., 2015). Nevertheless, our findings of diagnostic group differences suggest such interpersonal movement coordination metrics may be a promising approach for further analysis of dyadic caregiver-child interaction in very young children with and without ASD. These results further demonstrate the feasibility of applying video-derived movement analysis with young children (e.g., Pfeiffer et al., 2018). Future studies could examine interpersonal coordination during other types of play activities in young children, including during more natural settings (e.g., during a circle time activity on the floor or when explicitly instructed to use song-associated gestures), to further probe how context impacts interpersonal coordination in children with and without ASD.

The current study is novel in its consideration of both child and caregiver behavior in matched musical and non-musical natural interaction contexts. To our knowledge, this is the first study to compare caregiver-child engagement in a book sharing task across musical and nonmusical contexts and to consider how such contexts might impact both child and caregiver behavior in dyads with children with and without ASD. However, there are several limitations to consider. Although study participation did not require any musical skills, participants responded to study recruitment materials about musical activities and therefore may have a predisposition for music. Both the ASD and TD groups were heterogeneous in age and language level, and while matched on nonverbal developmental level, the ASD group had lower language skills than 


\section{PARENT-CHILD NONVERBAL ENGAGEMENT}

the TD group. There were individual differences in children's attention to the activities, which were related to language level of the ASD group. The small sample size may have precluded detection of significant effects; however, diagnostic differences were generally similar to those in a recent study of parent-child book sharing with a slightly larger sample size (Fleury \& Hugh, 2018). Future studies could include a larger sample size including a language age-matched group to further assess individual differences across contexts. Another key limitation is that dyads completed the picture book activity prior to completing the song book activity. Future studies could counterbalance tasks to control for any order effects. Nevertheless, as one might expect engagement and attention to decrease over time (e.g., Cirelli et al., 2020), our finding of increased visual attention during the song book activity (which was administered second), is consistent with the idea that attributes of the song book activity supported caregiver and child engagement in the activity and with each other as participants were already comfortable in the assessment settings prior to completing the book sharing activity. Finally, the nature of our movement analyses required that the caregiver and child sit across from each other at a table. Future studies may explore other measures of movement coordination that would allow for more natural positioning and touch during a caregiver-child book sharing activity, such as motion capture.

We examined child and caregiver attention and interaction during a book sharing activity. We found differences between young children with TD and ASD in their visual gaze behavior and interpersonal movement coordination, as well as differences in the visual gaze behavior of both children and parents during a musical context. Although prior studies have focused on how musical activities affect behavior of children with ASD, the current study highlights the importance of considering both partners in the interaction. Future studies can further investigate 
PARENT-CHILD NONVERBAL ENGAGEMENT

mechanisms by which musical contexts incidentally support caregiver-child engagement and how such activities are incorporated into therapeutic activities for families of children with ASD. Our findings indicate that musical activities can strengthen engagement between caregivers and children with ASD and TD.

\section{Declaration of Conflicting Interests}

The authors have no conflicts of interest to disclose. 
PARENT-CHILD NONVERBAL ENGAGEMENT

\section{References}

American Psychiatric Association. (2013). Diagnostic and statistical manual of mental disorders (Fifth edition). American Psychiatric Association.

https://doi.org/10.1176/appi.books.9780890425596

Bakeman, R. (2005). Recommended effect size statistics for repeated measures designs. Behavior Research Methods, 37(3), 379-384. https://doi.org/10.3758/BF03192707

Baron-Cohen, S. (1989). Joint-attention deficits in autism: Towards a cognitive analysis. Development and Psychopathology, 1(3), 185-189. https://doi.org/10.1017/S0954579400000377

Bates, D., Mächler, M., Bolker, B., \& Walker, S. (2015). Fitting linear mixed-effects models using lme4. Journal of Statistical Software, 67(1), 1-48. https://doi.org/10.18637/jss.v067.i01

Bean, A. F., Perez, B. I., Dynia, J. M., Kaderavek, J. N., \& Justice, L. M. (2019). Book-reading engagement in children with autism and language impairment: Associations with emergent-literacy skills. Journal of Autism and Developmental Disorders. https://doi.org/10.1007/s10803-019-04306-4

Beck, S. L., \& Reiser, J. (2020). Non-random acts of kindness: Joint music making increases preschoolers' helping and sharing with an adult. Psychology of Music. https://doi.org/10.1177/0305735620978688

Boorom, O., Muñoz, V., Xin, R., Watson, M., \& Lense, M. D. (2020). Parental responsiveness during musical and non-musical engagement in preschoolers with ASD. Research in Autism Spectrum Disorders, 78. https://doi.org/10.1016/j.rasd.2020.101641 


\section{PARENT-CHILD NONVERBAL ENGAGEMENT}

Cirelli, L. K., Einarson, K. M., \& Trainor, L. J. (2014). Interpersonal synchrony increases prosocial behavior in infants. Developmental Science, 17(6), 1003-1011. https://doi.org/10.1111/desc.12193

Cirelli, L. K., Jurewicz, Z. B., \& Trehub, S. E. (2020). Effects of maternal singing style on mother-infant arousal and behavior. Journal of Cognitive Neuroscience, 32(7), 1213 1220. https://doi.org/10.1162/jocn_a_01402

Cirelli, L. K., \& Trehub, S. E. (2019). Dancing to Metallica and Dora: Case study of a 19-monthold. Frontiers in Psychology, 10. https://doi.org/10.3389/fpsyg.2019.01073

Cirelli, L. K., \& Trehub, S. E. (2020). Familiar songs reduce infant distress. Developmental Psychology, 56(5), 861-868. https://doi.org/10.1037/dev0000917

Cirelli, L. K., Trehub, S. E., \& Trainor, L. J. (2018). Rhythm and melody as social signals for infants. Annals of the New York Academy of Sciences. https://doi.org/10.1111/nyas.13580

Cohen, J. (2013). Statistical power analysis for the behavioral sciences. Routledge. https://www.taylorfrancis.com/books/9780203771587

Constantino, J. N., Kennon-McGill, S., Weichselbaum, C., Marrus, N., Haider, A., Glowinski, A. L., Gillespie, S., Klaiman, C., Klin, A., \& Jones, W. (2017). Infant viewing of social scenes is under genetic control and is atypical in autism. Nature, 547(7663), 340-344. https://doi.org/10.1038/nature22999

Crum, S. (2012). Mine! Random House Children's.

Crawford, M. J., Gold, C., Odell-Miller, H., Thana, L., Faber, S., Assmus, J., Bieleninik, Ł., Geretsegger, M., Grant, C., Maratos, A., Sandford, S., Claringbold, A., McConachie, H., Maskey, M., Mössler, K. A., Ramchandani, P., \& Hassiotis, A. (2017). International 
PARENT-CHILD NONVERBAL ENGAGEMENT

multicentre randomised controlled trial of improvisational music therapy for children with autism spectrum disorder: TIME-A study. Health Technology Assessment, 21(59), 1-40. https://doi.org/10.3310/hta21590

Cryer, J. D., \& Chan, K. S. (2008). Time series analysis: With applications in R. Springer Science \& Business Media.

Davis, J. (2011). You are my sunshine. Scholastic Inc.

Day, A. (2009). Good dog, Carl. Baker \& Taylor, CATS.

Dean, R. T., \& Dunsmuir, W. T. M. (2016). Dangers and uses of cross-correlation in analyzing time series in perception, performance, movement, and neuroscience: The importance of constructing transfer function autoregressive models. Behavior Research Methods, 48(2), 783-802. https://doi.org/10.3758/s13428-015-0611-2

Eerola, T., Luck, G., and Toiviainen, P. (2006). An investigation of preschoolers' corporeal synchronization with music. Proceedings of the 9th International Conference on Music Perception and Cognition, Bologna, 472-476.

Endedijk, H. M., Ramenzoni, V. C. O., Cox, R. F. A., Cillessen, A. H. N., Bekkering, H., \& Hunnius, S. (2015). Development of interpersonal coordination between peers during a drumming task. Developmental Psychology, 51(5), 714-721. https://doi.org/10.1037/a0038980

Fancourt, D., \& Perkins, R. (2018). The effects of mother-infant singing on emotional closeness, affect, anxiety, and stress hormones: Music \& Science. https://doi.org/10.1177/2059204317745746 


\section{PARENT-CHILD NONVERBAL ENGAGEMENT}

Fitzpatrick, P., Diorio, R., Richardson, M. J., \& Schmidt, R. C. (2013). Dynamical methods for evaluating the time-dependent unfolding of social coordination in children with autism. Frontiers in Integrative Neuroscience, 7. https://doi.org/10.3389/fnint.2013.00021

Fitzpatrick, P., Romero, V., Amaral, J. L., Duncan, A., Barnard, H., Richardson, M. J., \& Schmidt, R. C. (2017). Social motor synchronization: Insights for understanding social behavior in autism. Journal of Autism and Developmental Disorders, 47(7), 2092-2107. https://doi.org/10.1007/s10803-017-3124-2

Fleury, V. P., \& Hugh, M. L. (2018). Exploring engagement in shared reading activities between children with autism spectrum disorder and their caregivers. Journal of Autism and Developmental Disorders, 48(10), 3596-3607. https://doi.org/10.1007/s10803-018-36328

Fournier, K. A., Hass, C. J., Naik, S. K., Lodha, N., \& Cauraugh, J. H. (2010). Motor coordination in autism spectrum disorders: A synthesis and meta-analysis. Journal of Autism and Developmental Disorders, 40(10), 1227-1240. https://doi.org/10.1007/s10803-010-0981-3

Giorgino, T. (2009). Computing and visualizing dynamic time warping alignments in R: The dtw package. Journal of Statistical Software, 31(7), 1-24.

Gulsrud, A. C., Hellemann, G., Shire, S., \& Kasari, C. (2016). Isolating active ingredients in a parent-mediated social communication intervention for toddlers with autism spectrum disorder. Journal of Child Psychology and Psychiatry, 57(5), 606-613. https://doi.org/10.1111/jcpp.12481 


\section{PARENT-CHILD NONVERBAL ENGAGEMENT}

Hardy, M. W., \& LaGasse, A. B. (2013). Rhythm, movement, and autism: Using rhythmic rehabilitation research as a model for autism. Frontiers in Integrative Neuroscience, 7. https://doi.org/10.3389/fnint.2013.00019

Hernandez-Ruiz, E. (2019). Feasibility of parent coaching of music interventions for children with autism spectrum disorder. Music Therapy Perspectives. https://doi.org/10.1093/mtp/miz016

Hernandez-Ruiz, E. (2020). Parent coaching of music interventions for children with ASD: A conceptual framework. Nordic Journal of Music Therapy, 29(3), 200-221. https://doi.org/10.1080/08098131.2019.1647447

Kern, P., Wolery, M., \& Aldridge, D. (2007). Use of songs to promote independence in morning greeting routines for young children with autism. Journal of Autism and Developmental Disorders, 37(7), 1264-1271. https://doi.org/10.1007/s10803-006-0272-1

Kim, J., Wigram, T., \& Gold, C. (2008). The Effects of improvisational music therapy on joint attention behaviors in autistic children: A randomized controlled study. Journal of Autism and Developmental Disorders, 38(9), 1758. https://doi.org/10.1007/s10803-008-0566-6

Kirschner, S., \& Tomasello, M. (2009). Joint drumming: Social context facilitates synchronization in preschool children. Journal of Experimental Child Psychology, 102(3), 299-314. https://doi.org/10.1016/j.jecp.2008.07.005

Klimenko, M. A. (2007). Interactional synchrony between mother and toddler during book reading. [Unpublished master's thesis]. University of Georgia.

Kokal, I., Engel, A., Kirschner, S., \& Keysers, C. (2011). Synchronized drumming enhances activity in the caudate and facilitates prosocial commitment — if the rhythm comes easily. PLOS ONE, 6(11), e27272. https://doi.org/10.1371/journal.pone.0027272 


\section{PARENT-CHILD NONVERBAL ENGAGEMENT}

LaGasse, A. B. (2014). Effects of a music therapy group intervention on enhancing social skills in children with autism. Journal of Music Therapy, 51(3), 250-275. https://doi.org/10.1093/jmt/thu012

Landry, S. H., Smith, K. E., \& Swank, P. R. (2006). Responsive parenting: Establishing early foundations for social, communication, and independent problem-solving skills. Developmental Psychology, 42(4), 627-642. https://doi.org/10.1037/0012-1649.42.4.627

Lanter, E., Freeman, D., \& Dove, S. (2013). Procedural and conceptual print-related achievements in young children with autism spectrum disorders. Focus on Autism and Other Developmental Disabilities, 28(1), 14-25. https:/doi.org/10.1177/1088357612459270

Leclère, C., Viaux, S., Avril, M., Achard, C., Chetouani, M., Missonnier, S., \& Cohen, D. (2014). Why synchrony matters during mother-child interactions: A systematic review. PLOS ONE, 9(12), e113571. https://doi.org/10.1371/journal.pone.0113571

Lense, M. D., \& Camarata, S. (2020). PRESS-Play: Musical engagement as a motivating platform for social interaction and social play in young children with ASD. Music \& Science, 3, 2059204320933080. https://doi.org/10.1177/2059204320933080

Lense, M. D., \& Jones, W. (2016, July). Beat-based entrainment during infant-directed singing supports social engagement. Proceedings of the 14th International Conference on Music Perception \& Cognition (ICMPC14), San Francisco, CA.

Lord, C., Rutter, M., DiLavore, P. C., Risi, S., Gotham, K., \& Bishop, S. (2012). Autism diagnostic observation schedule, second edition (ADOS-2). Western Psychological Services. 


\section{PARENT-CHILD NONVERBAL ENGAGEMENT}

Marsh, K. L., Isenhower, R. W., Richardson, M. J., Helt, M., Verbalis, A. D., Schmidt, R. C., \& Fein, D. (2013). Autism and social disconnection in interpersonal rocking. Frontiers in Integrative Neuroscience, 7. https://doi.org/10.3389/fnint.2013.00004

Matthews, T. E., Witek, M. A. G., Lund, T., Vuust, P., \& Penhune, V. B. (2020). The sensation of groove engages motor and reward networks. NeuroImage, 214, 116768. https://doi.org/10.1016/j.neuroimage.2020.116768

McGlensey, M. (2016, February 3). 16 People with autism describe why eye contact can be difficult. The Mighty. http://themighty.com/2016/02/why-eye-contact-can-be-difficultfor-people-with-autism/

McNaughton, K. A., \& Redcay, E. (2020). Interpersonal synchrony in autism. Current Psychiatry Reports, 22(3), 12. https://doi.org/10.1007/s11920-020-1135-8

Miller, J. E., Carlson, L. A., \& McAuley, J. D. (2013). When what you hear influences when you See: Listening to an auditory rhythm influences the temporal allocation of visual attention. Psychological Science, 24(1), 11-18. https://doi.org/10.1177/0956797612446707

Mullen, E. M. (1995). Mullen Scales of Early Learning. American Guidance Service Inc. Mundy, P. (2018). A review of joint attention and social-cognitive brain systems in typical development and autism spectrum disorder. The European Journal of Neuroscience, 47(6), 497-514. https://doi.org/10.1111/ejn.13720

Paul, A., Sharda, M., Menon, S., Arora, I., Kansal, N., Arora, K., \& Singh, N. C. (2015). The effect of sung speech on socio-communicative responsiveness in children with autism spectrum disorders. Frontiers in Human Neuroscience, 9. https://doi.org/10.3389/fnhum.2015.00555 


\section{PARENT-CHILD NONVERBAL ENGAGEMENT}

Pfeiffer, R., Wallace, M., \& Lense, M. (2018). Social motor coordination during adult-child interactions. Annals of the New York Academy of Sciences. https://doi.org/10.1111/nyas.13651

Politimou, N., Stewart, L., Müllensiefen, D., \& Franco, F. (2018). Music@Home: A novel instrument to assess the home musical environment in the early years. PloS One, 13(4), e0193819. https://doi.org/10.1371/journal.pone.0193819

Ramseyer, F. T. (2020). Motion energy analysis (MEA): A primer on the assessment of motion from video. Journal of Counseling Psychology, 67(4), 536. https://doi.org/10.1037/cou0000407

R Core Team. (2019). R: A language and environment for statistical computing. R Foundation for Statistical Computing. https://www.R-project.org

Raffi. (1988). Wheels on the Bus. Crown.

Sasson, N. J., \& Touchstone, E. W. (2014). Visual attention to competing social and object images by preschool children with autism spectrum disorder. Journal of Autism and Developmental Disorders, 44(3), 584-592. https://doi.org/10.1007/s10803-013-1910-z

Savage, P. E., Loui, P., Tarr, B., Schachner, A., Glowacki, L., Mithen, S., \& Fitch, W. T. (2020). Music as a coevolved system for social bonding. Behavioral and Brain Sciences, 1-36. https://doi.org/10.1017/S0140525X20000333

Schultz, B. G., O'Brien, I., Phillips, N., McFarland, D. H., Titone, D., \& Palmer, C. (2016). Speech rates converge in scripted turn-taking conversations. Applied Psycholinguistics, 37(5), 1201. https://doi.org/10.1017/S0142716415000545 


\section{PARENT-CHILD NONVERBAL ENGAGEMENT}

Schultz, B. G., Brown, R. M., \& Kotz, S. A. (2021). Dynamic acoustic salience evokes motor responses. Cortex, 134, 320-332. https://doi.org/10.1016/j.cortex.2020.10.019

Schultz, B. G., \& Palmer, C. (2019). The roles of musical expertise and sensory feedback in beat keeping and joint action. Psychological Research, 83(3), 419-431. https://doi.org/10.1007/s00426-019-01156-8

Shenfield, T., Trehub, S. E., \& Nakata, T. (2003). Maternal singing modulates infant arousal. Psychology of Music, 31(4), 365-375. https://doi.org/10.1177/03057356030314002

Singmann, H., Bolker, B., Westfall, J., Aust, F., \& Ben-Shachar, M. S. (2020). afex: Analysis of factorial experiments. R package version 0.27-2. https:/CRAN.Rproject.org/package $=$ afex

Simpson, K., Keen, D., \& Lamb, J. (2013). The use of music to engage children with autism in a receptive labelling task. Research in Autism Spectrum Disorders, 7(12), 1489-1496. https://doi.org/10.1016/j.rasd.2013.08.013

Stephenson, L. J., Edwards, S. G., \& Bayliss, A. P. (2021). From gaze perception to social cognition: The shared-attention system. Perspectives on Psychological Science, 16(3), 553-576. https://doi.org/10.1177/1745691620953773

Stern, D. N. (1974). Mother and infant at play: The dyadic interaction involving facial, vocal, and gaze behaviors. In The effect of the infant on its caregiver (pp. xxiv, 264-xxiv, 264). Wiley-Interscience.

Tapp, J., \& Walden, T. (1993). PROCODER: A professional tape control, coding, and analysis system for behavioral research using videotape. Behavior Research Methods, Instruments, \& Computers, 25(1), 53-56. https://doi.org/10.3758/BF03204449 


\section{PARENT-CHILD NONVERBAL ENGAGEMENT}

Tarr, B., Launay, J., \& Dunbar, R. I. M. (2014). Music and social bonding: "Self-other” merging and neurohormonal mechanisms. Frontiers in Psychology, 5. https://doi.org/10.3389/fpsyg.2014.01096

Thompson, G. A. (2018). Long-term perspectives of family quality of life following music therapy with young children on the autism spectrum: A phenomenological study. Journal of Music Therapy, 54(4), 432-459. https://doi.org/10.1093/jmt/thx013

Thompson, G. A., \& Abel, L. A. (2018). Fostering spontaneous visual attention in children on the autism spectrum: A proof-of-concept study comparing singing and speech. Autism Research, 11(5), 732-737. https://doi.org/10.1002/aur.1930

Thompson, G. A., Shanahan, E. C., \& Gordon, I. (2019). The role of music-based parent-child play activities in supporting social engagement with children on the autism spectrum: A content analysis of parent interviews. Nordic Journal of Music Therapy, 28(2), 108-130. https://doi.org/10.1080/08098131.2018.1509107

Trainor, L. J., \& Cirelli, L. (2015). Rhythm and interpersonal synchrony in early social development: Interpersonal synchrony and social development. Annals of the New York Academy of Sciences, 1337(1), 45-52. https://doi.org/10.1111/nyas.12649

Trehub, S. E., Plantinga, J., \& Russo, F. A. (2016). Maternal vocal interactions with infants: Reciprocal visual influences: Maternal speech and singing. Social Development, 25(3), 665-683. https://doi.org/10.1111/sode.12164

Trevarthen, C., \& Aitken, K. J. (2001). Infant intersubjectivity: Research, theory, and clinical applications. Journal of Child Psychology and Psychiatry, 42(1), 3-48. https://doi.org/10.1111/1469-7610.00701 


\section{PARENT-CHILD NONVERBAL ENGAGEMENT}

Tunçgenç, B., \& Cohen, E. (2018). Interpersonal movement synchrony facilitates pro-social behavior in children's peer-play. Developmental Science, 21(1), e12505. https://doi.org/10.1111/desc.12505

Westerveld, M. F., Paynter, J., \& Wicks, R. (2020). Shared book reading behaviors of parents and their verbal preschoolers on the autism spectrum. Journal of Autism and Developmental Disorders. https://doi.org/10.1007/s10803-020-04406-6

Wimpory, D. C., Hobson, R. P., \& Nash, S. (2007). What facilitates social engagement in preschool children with autism? Journal of Autism and Developmental Disorders, 37(3), 564-573. https://doi.org/10.1007/s10803-006-0187-x

Zampella, C. J., Csumitta, K. D., Simon, E., \& Bennetto, L. (2020). Interactional synchrony and its association with social and communication ability in children with and without autism spectrum disorder. Journal of Autism and Developmental Disorders.

https://doi.org/10.1007/s10803-020-04412-8 
PARENT-CHILD NONVERBAL ENGAGEMENT

Figure 1

Child Mean Visual Attention Coding (VAC) Scores by Diagnostic Group and Book Activity

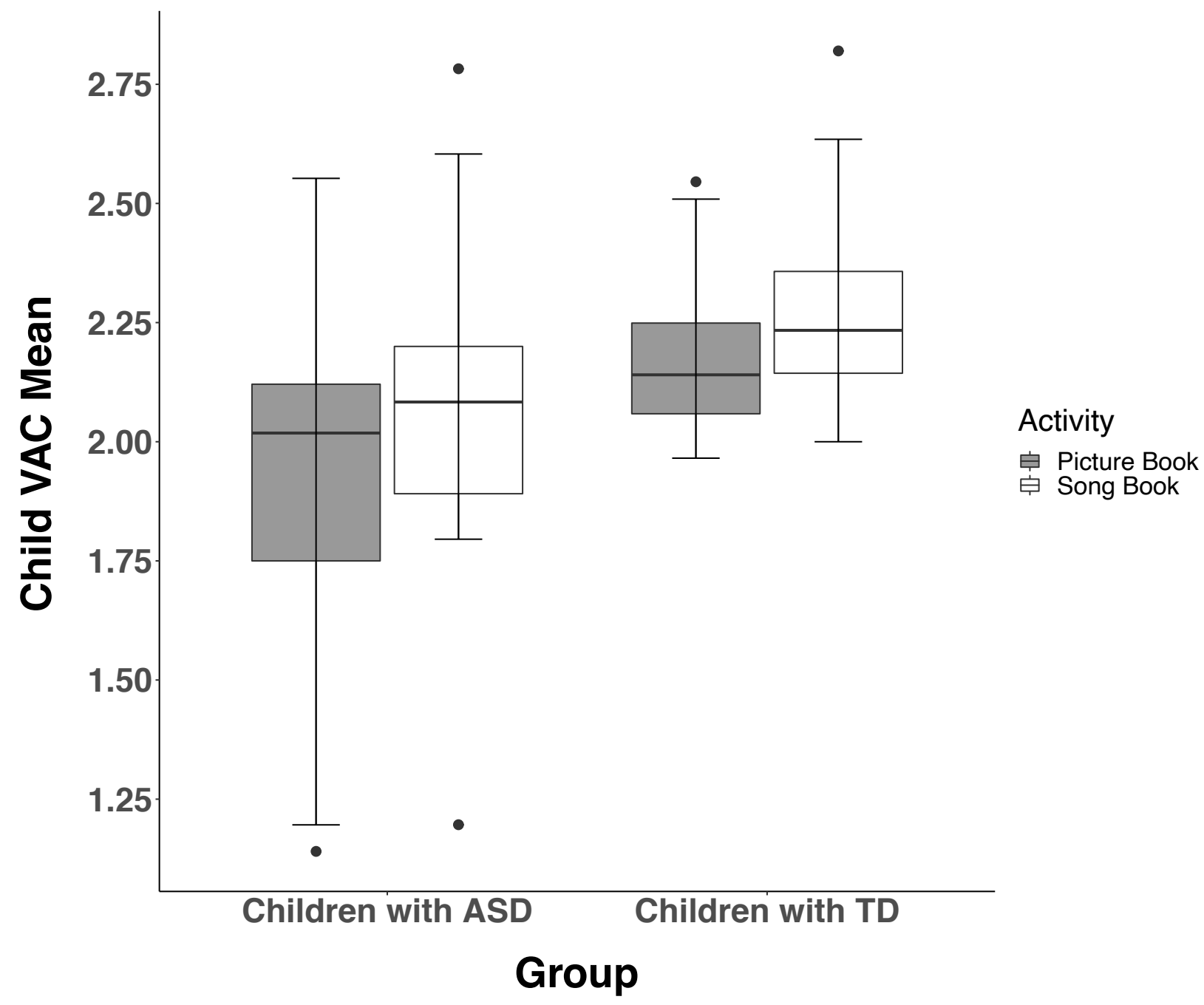


PARENT-CHILD NONVERBAL ENGAGEMENT

Figure 2

Caregiver Mean Visual Attention Coding (VAC) Scores by Diagnostic Group and Book Activity

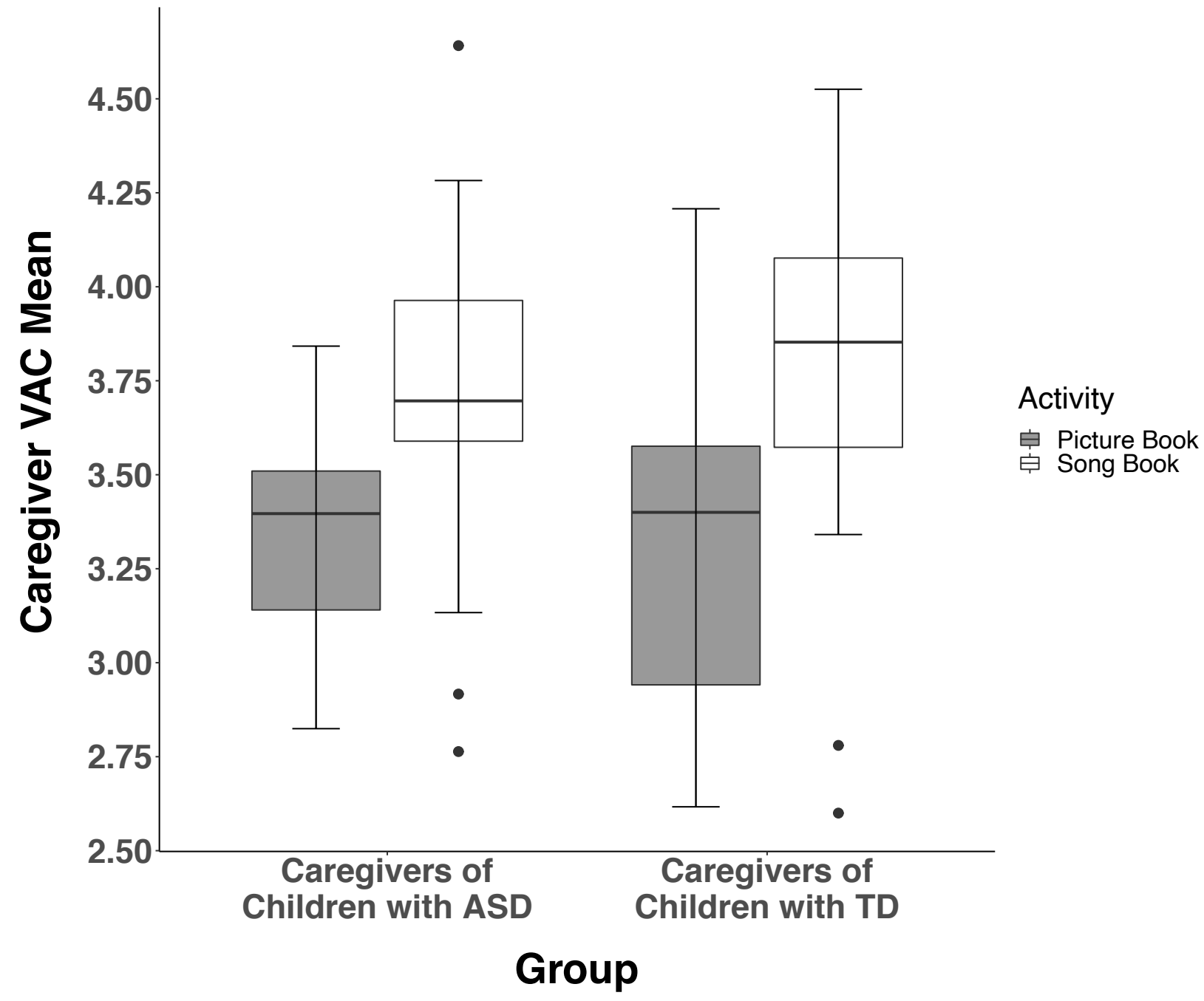


PARENT-CHILD NONVERBAL ENGAGEMENT

\section{Figure 3}

Relationship Between Caregiver and Child Visual Attention Coding (VAC) Scores for Each Book Activity by Diagnostic Group

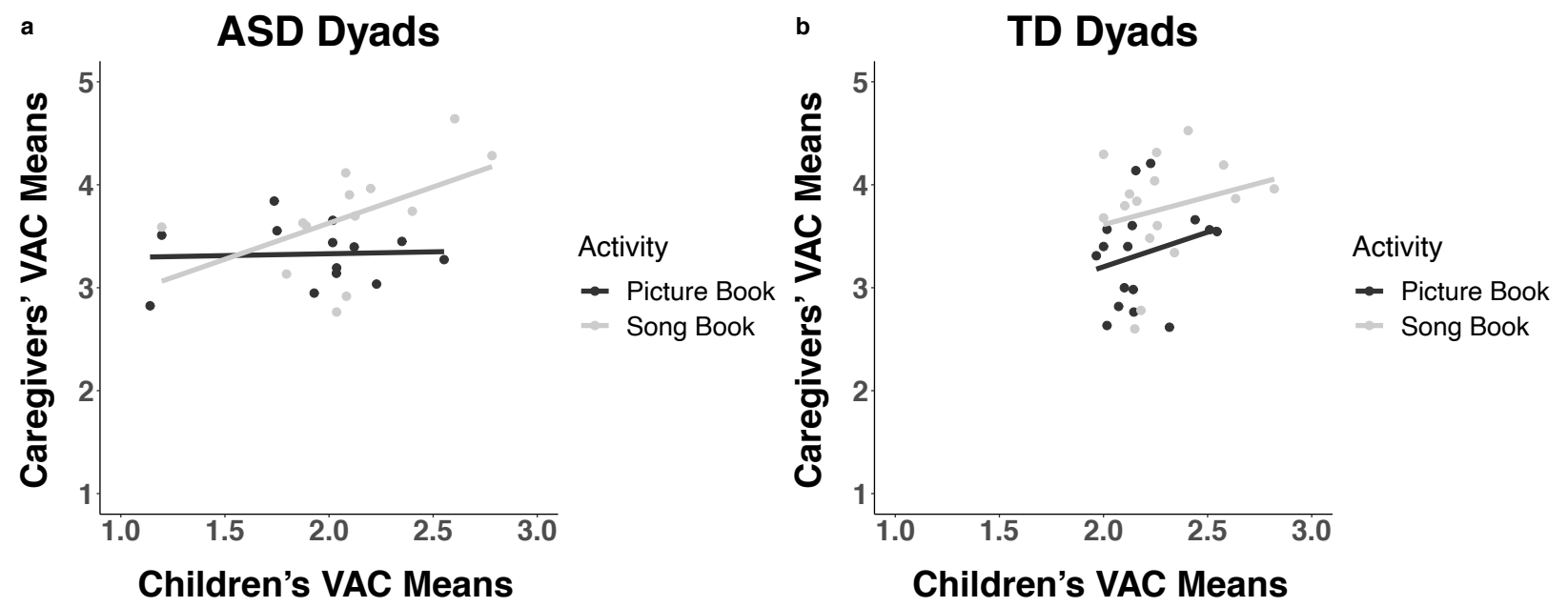

Note. Relationship between caregiver and child visual attention coding (VAC) scores for each book activity in dyads with children with ASD (3a) and with TD (3b). 
PARENT-CHILD NONVERBAL ENGAGEMENT

Figure 4

Interpersonal Movement Coordination Cross-Correlations by Diagnostic Group and Book Activity

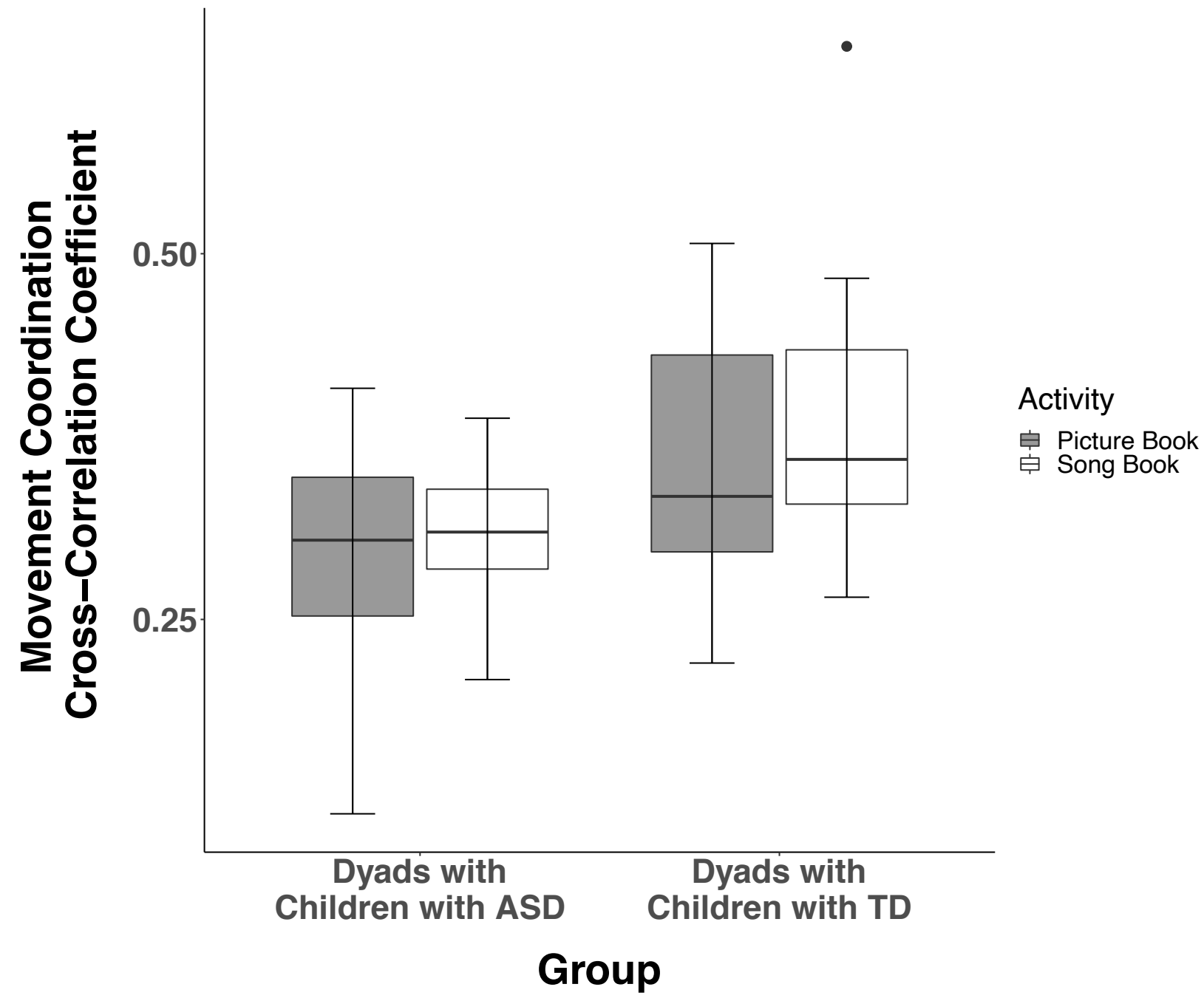

